\title{
DIAGNÓSTICO IN VIVO DA SENSIBILIDADE DE NEMATOIDES A DIFERENTES ANTIHELMÍNTICOS EM OVINOS CRIADOS EM SISTEMA SEMI-INTENSIVO
}

(In vivo diagnostic sensitivity of nematodes to different anthelmintics in sheep reared in semi-intensive system)

Renan Zappavigna Costa Starling; Isabella Vilhena Martins ${ }^{1}$, Isabella Vilhena Martins, Cintia Silva Alves, Marcos Vinícius Gonçalves Viana, Willy Stefanon Dietrich

1Correspondência: ivfmartins@gmail.com

RESUMO: Grande variação no grau de resistência de populações de nematódeos é observada em grupos químicos, entre eles os anti-helmínticos, sendo este o principal entrave para obtenção do controle da verminose na ovinocultura. $O$ objetivo do estudo foi avaliar a sensibilidade de nematoides de ovinos mestiços em criação semi-intensiva em três propriedades a cinco princípios ativos (levamisol, albendazol, ivermectina, monepantel e closantel) utilizados nas vermifugação. Foram analisadas amostras fecais, em três momentos (antes, 7 e 14 dias após o tratamento) utilizando a técnica de Gordon e Withlock (OPG), processadas e calculadas pelas fórmulas Eficácia=(1-[OPGtratado/OPGcontrole])x100 e Redução de OPG $=100 \times$ [OPGto - OPGtn/OPGto ]. Nos momentos sete e quatorze os grupos tratamentos diferiram significativamente $(p<0,05 \%)$ do momento zero, porém, os animais tratados com ivermectina no momento quatorze não apresentaram diferença significativa quando comparado ao grupo controle. No exame coproparasitológico os gêneros mais frequentes foram Haemonchus e Trichostrongylus. $\mathrm{Na}$ primeira propriedade $\mathrm{o}$ monepantel $\mathrm{e} O \mathrm{o}$ albendazol demonstraram os melhores resultados de redução de OPG, na segunda propriedade o levamisol, closantel e monepantel apresentaram resultados expressivos quando comparado aos demais fármacos e na terceira propriedade os fármacos que tiveram melhores resultados de redução de OPG foram levamisol e monepantel. Nas propriedades A, B e C, foram diagnosticadas populações de nematoides resistentes a ivermectina. Nas propriedades $B$ e $C$, foram diagnosticadas populações de nematoides resistentes a albendazol.

Palavras-chave: ruminantes; resistência; helmintos

ABSTRACT: Wide variation in the degree of resistance to nematode populations is observed in chemical groups, including the anthelmintics, which is the main obstacle to obtaining control of worms in the sheep industry. The aim of the study was to evaluate the sensitivity of nematodes of crossbred sheep in semi-intensive farming in three properties to levamisole, albendazole, ivermectin, closantel and monepantel) used in worming. Fecal samples were analyzed at three different times (before, 7 and 14 days after treatment) using the technique Gordon and Whitlock (EPG) processed and calculated by the formulas Efficacy $=(1-$ [OPGtreat $/$ OPGcontrol]) $\times 100$ and the formula Reduction OPG $=100 \times$ [OPGt0 - OPGtn / OPGt0]. After the treatment groups differed significantly $(p<0.05 \%)$ from before, however, animals treated with ivermectin in the after fourteenth days showed no significant difference when compared to the control group. In the fecal examinations the most common genera were Haemonchus and Trichostrongylus. In the first property, monepantel and albendazole showed the best EPG reduction results, the second property levamisole, closantel and monepantel showed significant results when compared to other drugs and in the third property the better EPG reduction results were levamisole and monepantel. In the properties $A, B$ and $C$, resistant populations of nematodes to ivermectin were diagnosed. $\mathrm{B}$ and $\mathrm{C}$ on the properties, for nematode populations resistant to albendazole were diagnosed.

Key Words: ruminant; resistance; helminths 


\section{INTRODUÇÃO}

O principal fator limitante da ovinocultura mundial, especificamente em regiões tropicais, são os casos de endoparasitoses e 0 baixo conhecimento sobre a resistência parasitária na criação semi-intensivo de ovinos, onde os prejuízos econômicos são acentuados. Estima-se que mais de $60 \%$ das perdas econômicas na ovinocultura são decorrentes da ação de parasitos (Costa et al., 2011). Como consequência da existência do parasitismo sobre o rebanho, é notório o retardo no crescimento, a redução dos parâmetros produtivos e até morte de indivíduos jovens.

O uso indiscriminado de produtos químicos no controle desses parasitos gastrointestinais tem favorecido 0 aparecimento da resistência antihelmíntica em vários países (Kaplan e Vidyashankar, 2012) incluindo o Brasil. Segundo Fortes et al.(2013), a seleção e o crescimento de populações resistentes aos grupos de antihelmínticos, avermectinas, imidotiazóis e benzimidazóis, constituem um sério obstáculo a ovinocultura e o monitoramento de populações resistentes se tornam imprescindíveis, principalmente, nas decisões relacionadas ao controle parasitário no rebanho.

No entanto, não há na literatura pesquisada estudos que discorram sobre o diagnóstico da resistência antihelmíntica em nematóides de ovinos no Estado do Espírito Santo. Assim, o objetivo deste trabalho foi avaliar a sensibilidade de nematóides a diferentes anti-helmínticos, de ovinos criados em sistema semi-intensivo no Estado do Espírito Santo.

\section{MATERIAL E MÉTODOS}

Foram utilizados 158 animais mestiços, com idade variando de três a
18 meses, independente de sexo e fase produtiva, sem tratamento prévio com anti-helmíntico por um período mínimo de 30 dias e com a contagem de ovos por grama de fezes (OPG) superior a 300 ovos. Os animais recebiam ração concentrada no cocho pela manhã, sal mineral para ovinos, pastejo no decorrer do dia (Brachiaria sp.) e água ad libitum. Os animais foram previamente identificados por meio de brincos ou colares coloridos e divididos em seis grupos de 10 animais, sendo um grupo controle e cinco tratados, com diferentes anti-helmínticos.

Os animais foram medicados via oral nos seguintes grupos: Grupo 1 tratamento com Cloridrato de levamisol a $5 \%(5 \mathrm{mg} / \mathrm{kg})$; Grupo 2 - tratamento com ivermectina a $1 \%(0,2 \mathrm{mg} / \mathrm{kg})$; Grupo 3 - tratamento com closantel à $10 \%(10 \mathrm{mg} / \mathrm{kg})$; Grupo 4 - tratamento com Sulfóxido de albendazole $(1 \mathrm{~mL} / 20$ $\mathrm{kg}$ ); Grupo 5 - tratamento com monepantel (2,5 mg/kg); Grupo 6 (GC) grupo controle, sem tratamento.

As amostras de fezes para análise de ovos de helmintos gastrointestinais foram coletadas de todos os animais, individualmente diretamente da ampola retal, utilizando-se sacos plásticos, devidamente identificados e mantidos sobre refrigeração em caixa térmica com gelo. As coletas foram realizadas em três momentos, dia 0 - antes do tratamento (M0), dia 7 (M7) e dia 14 (M14) após tratamento.

No M0, após a coleta das amostras e realização do OPG, os animais foram selecionados e divididos aleatoriamente nos grupos (GC, G1, G2, G3, G4 e G5). Os animais foram pesados individualmente por meio de fita de pesagem e medicados com 0 princípio ativo do respectivo grupo na dosagem estabelecida pelo fabricante.

Após a coleta, as amostras foram encaminhadas ao laboratório de Parasitologia do Hospital Veterinário (HOVET) da Universidade Federal do 
Espírito Santo e processadas pela técnica Macmaster (Gordon e Whitlock,1939) para contagem de ovos por grama de fezes (OPG).

Foi realizada ainda a coprocultura com as amostras pela técnica de Roberts e O'Sullivan (1950). Após sete dias de incubação as larvas eclodidas foram colocadas em tubos falcon com capacidade para $15 \mathrm{~mL}$ e acondicionadas sob refrigeração até preparação e leitura das lâminas. Das larvas obtidas na coprocultura realizouse a identificação genérica de 100 larvas para estimativa de composição da carga parasitária, conforme a descrição de Keith (1953).

Para análise estatística dentro das propriedades os dados foram tabulados em planilha do Microsoft Excel 2007, e analisados por estatística descritiva mediante ao cálculo de eficácia a campo e redução de OPG. Por meio dos valores de OPG de cada grupo, foi calculada a média, em cada momento, e com esses valores pôde-se calcular a redução da contagem de ovos nas fezes, e a eficácia de cada fármaco testado. $\mathrm{Na}$ análise descritiva dos dados, obtiveram-se valores da média e do desvio padrão de cada grupo.

De acordo com as recomendações da World Association for the Advancement of Veterinary Parasitology (WAAVP) (Coles et al., 1992), foi realizado o teste de redução na contagem de ovos por grama de fezes (TRCOF) que determina a eficácia dos princípios ativos testados, levando-se em consideração o OPG do grupo controle. Para determinar a redução da contagem de ovos por grama de fezes foi utilizado fórmula descrita por Coles et al. (1992): Eficácia = $100 \times 1$ - (OPGt /OPGc)

$\mathrm{OPGt}=$ média do OPG do grupo tratado em cada momento (7 e 14); OPGc = média do OPG do grupo controle em cada momento (7 e 14).
A avaliação da eficácia dos fármacos utilizados baseou-se na determinação proposta pelo Grupo Mercado Comum (GMC) para antihelmínticos, sendo altamente efetivo quando reduz maior que $98 \%$ do OPG, efetivo com 90 - 98\%, moderadamente efetivo com $80-89 \%$ e insuficientemente ativo com menor que $80 \%$ de redução e não registrável (GMC, 1996).

O delineamento utilizado foi 0 inteiramente casualizado no esquema de parcelas subdividas $6 \times 3 \quad(5$ tratamentos + Controle $\times 3$ momentos). Os dados de OPG os dados foram submetido à análise de variância $(P<0,05)$, sendo as médias comparadas pelo teste de Tukey $(P<0,05)$, sendo os mesmo transformados por $\log _{10}(x+1)$. Para a realização das análises utilizou-se os pacotes Agricolae (Mendiburu, 2014) e ExpDes.pt (Ferreira et al., 2013) do aplicativo computacional $R$ versão 3.0 (R CORE TEAM, 2013).

\section{RESULTADOS E DISCUSSÃO}

Os exames coproparasitológicos dos animais avaliados apresentaram presença de ovos de helmintos da família Trichostrongylidae e também dos gêneros Strongyloides, Moniezia e oocistos de Eimeria. O percentual médio de gêneros de helmintos encontrados nos animais dos grupos amostrais foi em torno de $50 \%$ de Haemonchus e $50 \%$ de Trichostrongylus em duas propriedades estudadas, concordando com outros estudos no sul do Brasil (Morais et al., 2002). Em uma propriedade este percentual ficou acima de $90 \%$ para Haemonchus.

Analisando os dados por propriedades, a propriedade $A$ foi constatada interação significativa entre o OPG dos animais tratamentos e nos momentos (zero, sete e quatorze). Todavia, somente nos momentos sete e quatorze os grupos 
tratamentos diferiram significativamente do grupo controle ( $p<0,05 \%)$. No momento sete, o grupo tratado com monepantel foi o que apresentou o menor valor de OPG, diferindo-se do grupo controle. $O$ albendazol apresentou valor de OPG intermediário quando comparado com o monepantel e ivermectina. No momento quatorze, verificou-se que o grupo tratado com monepantel persistiu com o menor valor de OPG.

$\mathrm{Na}$ propriedade $\mathrm{B}$ foi constatada interação significativa entre o OPG dos animais tratamentos e nos momentos (zero, sete e quatorze), exceto a ivermectina no momento 14. Todavia, somente nos momentos sete e quatorze os grupos tratamentos diferiram significativamente $(p<0,05 \%)$. No momento sete, o grupo tratado com monepantel zerou o valor de OPG, diferindo-se do grupo controle, ivermectina e albendazol. O closantel apresentou valor de OPG intermediário quando comparado com o monepantel e ao levamisol. No momento quatorze, verificou-se que o grupo tratado com closantel zerou o valor de OPG, e o monepantel apresentou o valor de OPG intermediário.

$\mathrm{Na}$ propriedade $\mathrm{C}$ foi constatada interação significativa entre o OPG dos animais tratamentos e nos momentos (zero, sete e quatorze), para o closantel, monepantel e levamisol exceto no momento 14. Todavia, somente nos momentos sete e quatorze os grupos tratamentos diferiram significativamente $(p<0,05 \%)$. No momento sete, o grupo tratado com levamisol foi 0 que apresentou o menor valor de OPG, diferindo-se do grupo controle, ivermectina e albendazol. O monepantel apresentou valor de OPG intermediário quando comparado com levamisol e closantel. No momento quatorze, verificou-se que o grupo tratado com monepantel apresentou o menor valor de OPG.
Na propriedade A o monepantel e
- albendazol foram os que demonstraram os melhores resultados, com redução expressiva de OPG no momento sete e quatorze (Figura 1) nos dois momentos avaliados. Os animais tratados com o levamisol e ivermectina apresentaram uma eficácia da redução de ovos por grama de fezes moderada (80,06 - 89,9\% segundo GMC), sendo que o levamisol apresentou uma redução de OPG insuficientemente ativa $(80 \%)$, enquanto a ivermectina uma redução de OPG não registrável, tanto no momento sete $(25 \%)$ quanto no momento quatorze $(0 \%)$. Sendo assim, a ivermectina foi considerada nessa propriedade um anti-helmíntico ineficaz, visto que no questionário foi relatado 0 uso deste fármaco já na vermifugação desses animais por diversas vezes de forma indiscriminada, sendo este relato indicativo de resistência parasitária. 0 closantel neste estudo apresentou uma eficácia na redução de ovos por grama de fezes inferior a $80 \%$ e uma redução de OPG intermediaria quando comparada aos demais fármacos (acima de $85 \%$ ), o que pode ser observado na Figura 1. 


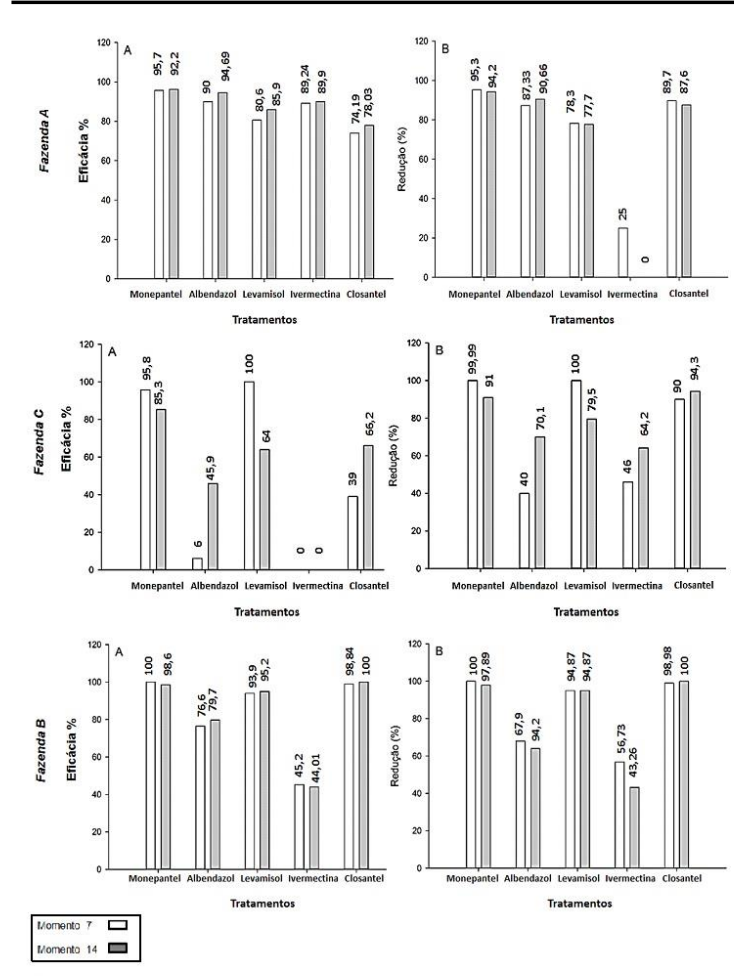

Figura 1. Valores de eficácia (A) e redução de $O P G(B)$, nos momentos 7 e 14 após o uso de anti-helmínticos no controle de nematoides gastrointestinais de ovinos, nas propriedades localizadas no Município de Alegre, Espírito Santo.

$\mathrm{Na}$ propriedade $\mathrm{B}$ os princípios ativos levamisol, closantel e monepantel apresentaram eficácia de 93,9; 98,84 e $100 \%$ respectivamente no momento sete, e 95,2; 100 e $98,6 \%$ respectivamente no momento quatorze. O grupo de animais tratado com albendazol resultou em eficácia de 76,66 e $79,7 \%$, respectivamente para os momentos sete e quatorze, e uma redução de OPG de $67,9 \%$ no momento sete e $64,2 \%$ para o momento quatorze. Quando avaliados os animais do grupo tratado com ivermectina observou-se baixa eficácia nos dois momentos $(45,23$ e $44,1 \%)$, respectivamente.

Ao avaliar a redução de OPG dos grupos tradados, verificou-se que, 0 levamisol teve redução de $94,87 \%$, nos dois momentos, considerado assim efetivo, enquanto o closantel apresentou-se altamente efetivo com valores de 98,98 e $100 \%$, e os animais tratados com monepantel no momento sete foi considerado altamente efetivo com $100 \%$ de redução, e no momento quatorze $97,89 \%$, classificado como efetivo. O grupo tratado com albendazol foi considerado de baixo valor de redução $(67,9$ e $64,2 \%$ no momento sete e quatorze respectivamente). Os animais vermifugados com ivermectina apresentaram valores muito baixo de redução nos dois momentos, quando comparado com os demais fármacos, sendo 56,73 e 43,26\% respectivamente, como demostrado também na Figura 1.

$\mathrm{Na}$ propriedade $\mathrm{C}$ o levamisol apresentou eficácia no momento sete de $100 \%$, decaindo no momento quatorze para 64\%, e redução de 100 e 79,5 respectivamente nos momentos sete e quatorze. O tratamento com monepantel apresentou eficácia de $95,8 \%$ de $85,3 \%$ nos momentos sete e quatorze, respectivamente e redução de OPG de $99,9 \%$ e $91 \%$, respectivamente, para os momentos sete e quatorze. Já os tratamentos com albendazol, ivermectina e closantel demostraram uma redução de OPG no momento sete de 45,$9 ; 46$ e $66,22 \%$ e no momento quatorze $(70,1 ; 64,2$ e $94,3 \%)$ respectivamente. Entretanto, quando avaliado os valores de eficácia no momento sete $(6 ; 0 ; 39 \%)$ e no momento quatorze $(40 ; 0 ; 90 \%)$ os mesmos demostraram valores muito baixos. Um estudo realizado na Venezuela, mais especificamente em Trinidad, apontou resistência de $46 \%$ e $61 \%$ do fármaco albendazol (George et al., 2011). A resistência dos helmintos em relação ao albendazol também foi registrada por Gill (1996), Soccol et al., (1996) e Molento (2004). Lima et al. (2010) relataram que nos rebanhos tratados com albendazol os percentuais de redução do OPG (30\% e 70,50\%) concluindo 0 indicativo de resistência, como observado na Figura 1.

Quanto à redução de ovos por grama de fezes (eficácia) e a redução de OPG em relação ao momento zero, de cada anti-helmíntico, os diferentes fármacos apresentaram níveis de eficácia distintos. Neste trabalho, nas 
propriedades $A, B$ e $C$ a ivermectina foi ineficaz, por não ter controlado a infecção de helmintos gastrointestinais dos animais tratados, uma vez que não houve diminuição da média de OPG dos animais do grupo tratado por este princípio ativo no momento quatorze. Mais especificamente à molécula Ivermectina, os resultados do presente estudo não diferem do descrito por outro autores, como Soccol et al (1996), Molento (2004) e Rosalinski-Moraes et al. (2007). Lima et al. (2010), no estado de Pernambuco observaram baixa eficácia da ivermectina com resultado de $67,33 \%$, em animais meio sangue, criados em manejo semi-intensivo. Outro estudo realizado por RosalinskiMorais et al. (2007) em animais sem ração definida, criados a pasto, relataram casos de resistência em 100\% da propriedade estudadas, e concluíram que este medicamento está sendo limitado, uma vez que o uso indiscriminado vem causando baixa eficácia não sendo justificado sua utilização. Sczesny-Morais et al. (2010) relataram no estado do Mato Grosso do Sul resistência a diversos princípios ativos, em ovinos meio sangue em criação semi-intensivo, com percentuais de eficácia de $0 \%$ para ivermectina.

Com relação ao albendazol, nas propriedades $\mathrm{B}$ e $\mathrm{C}$ o fármaco também foi ineficaz. Outros autores também relataram o problema no Brasil, como Sczesny-Morais et al. (2010) relataram no estado do Mato Grosso do Sul, encontrando $0,7 \%$ de eficácia para 0 albendazol. Lima et al. (2010) no estado de Pernambuco relataram resistência $0,7 \%$ de eficácia para albendazol. George et al. (2011), objetiva avaliar a eficácia de anti-helmínticos no controle de nematóides gastrointestinais de cordeiros e a resistência aos antihelmínticos em Trinidad, concluiu-se que o tratamento feito com albendazol e levamisol não foram eficazes.
Outros estudos revelaram índices de resistência mais baixos (Melo et al., 2003; Álvarez-Sánchez et al., 2006; Traversa et al., 2007; Köse et al., 2007) entretanto, considerando a situação desse fenômeno no mundo, pode-se dizer que existe tendência de aumento nos níveis de resistência parasitária pela persistência do uso deste princípio ativo, devido principalmente à cultura dos criadores em atribuir a mesma alta eficiência facilitada pela redução do preço dos produtos comerciais a base dessa formulação.

A partir desse estudo recomendase 0 uso dos anti-helmínticos específicos para cada propriedade e medidas de manejo auxiliares, visto que o manejo de vermifugação é realizado de formas distintas, e os resultados de eficácia foram diferentes entre os criatórios.

$\mathrm{Na}$ propriedade $\mathrm{A}$ recomenda-se a utilização do albendazol e monepantel, uma vez que os mesmos apresentaram uma redução de OPG efetiva (90-98\%). Nesta propriedade, todos os anti-helmínticos foram utilizados em seu histórico, sendo que o monepantel foi aplicado nas últimas vermifugações, de acordo com os parâmetros clínicos dos animais, e com os resultados de OPG realizados no rebanho. De acordo com o questionário respondido pelo gerente da propriedade são utilizadas práticas de manejo como rotação de pastagem, limpeza dos bebedouros, separação dos animais por faixa etária e retirada de fezes do aprisco, porém as fezes são jogadas diretamente na capineira, o que facilita a reinfecção desses animais. A rotação de áreas de pastagens é extremamente recomendável nos sistemas de produção de ovinos, pois contribui para a redução na população de larvas viáveis, porém deve-se estar atento a forma como é realizada o período de descanso, número de piquetes e tempo de pastejo devem ser estabelecidos em 
função da gramínea forrageira predominante na pastagem (Chagas et al., 2007), o que não foi relatado no questionário, mas poderia ser instituído como auxiliar no controle da verminose, já que se trata de uma propriedade com 88 hectares, sendo que para a criação de ovinos é utilizada apenas 1 hectar.

$\mathrm{Na}$ propriedade $\mathrm{B}$ recomenda-se a utilização do monepantel e closantel que foram altamente eficazes (> 98\%), sendo também recomentado o uso do fármaco levamisol, que apresentou valor de efetivo na redução de OPG (90$98 \%)$. Os fármacos utilizados até 0 momento foram albendazol, levamisol e closantel, sendo o último utilizado atualmente. De acordo com o questionário respondido pelo profissional responsável pela propriedade são utilizadas práticas de manejo como, limpeza dos bebedouros e retirada de fezes do aprisco quinzenalmente, porém as fezes também são jogadas diretamente na capineira, o que facilita a reinfecção desses animais. Essas práticas, segundo Amarante et al.(2004), são essenciais para 0 controle de verminoses na criação de ovinos, e fazse necessária a implantação de esterqueira para o tratamento dos dejetos obtidos da limpeza dos estábulos, o que não foi relatado pelo administrador da propriedade, mas poderia ser instituído como auxiliar no controle da verminose, já que se trata de uma propriedade com 360 hectares, sendo que para a criação de ovinos é utilizada em apenas 0,5 hectar da mesma.
$\mathrm{Na}$ propriedade $\mathrm{C}$ recomenda-se a utilização do monepantel, que apresentou grande capacidade de redução de OPG para os dois momentos estudados. Neste caso, foram relatados o uso dos fármacos ivermectina e albendazol, sendo 0 último utilizado atualmente. A partir do questionário respondido pelo zootecnísta responsável pelo criatório de ovinos dessa propriedade, algumas práticas de manejo são empregadas, como: limpeza de bebedouros, aprisco do rebanho de piso ripado, sendo o mesmo limpo quinzenalmente, porém as fezes são jogadas diretamente nas capineiras, proporcionando assim a facilidade da reinfecção, pela ingestão da forrageira.

$\mathrm{Na}$ região onde as propriedades estudadas estão localizadas, é comum a realização de práticas errôneas como jogar as fezes retiradas do aprisco diretamente na capineira, realizar troca rotineira de princípios ativos, não separação dos animais por idade, sendo esta última prática observada apenas em uma das propriedades estudadas. A possibilidade de integrar outras formas de controle, como o uso racional dos anti-helmínticos, realização de limpeza frequente do aprisco, utilização de esterqueiras, evitar superlotação das pastagens, uso de consorcio com outras espécies animais e realização de exames de OPG e Famacha, diminuem o uso dos fármacos contribuindo para que os problemas de resistência antihelmíntica sejam retardados.

\section{CONCLUSÃO}

Nas propriedades A, B e C, foram diagnosticadas populações de nematoides resistentes a ivermectina.

Nas propriedades B e C, foram diagnosticadas populações de nematoides resistentes a albendazol. 


\section{NOTAS INFORMATIVAS}

O projeto foi aprovado pelo comitê de ética (CEUA), sob o número 013/2014 da Universidade Federal do Espírito Santo.

\section{REFERÊNCIAS}

ÁLVAREZ-SÁNCHEZ, M.A., PÉREZGARCIA, J., CRUZ-ROJO, M.A. e ROJO-VÁSQUEZ, F.A. Anthelmintic resistance in trichostrongylid nematodes of sheep farms in Northwest Spain. Parasitology Research, v. 99, p. 78-83, 2006.

AMARANTE, A.F.T.; BRICARELLO, P.A.; ROCHA, R.A.; GENNARI, S.M. Resistance of Santa Ines, Suffolk and lle de France lambs to naturally acquired gastrointestinal nematode infections. Veterinary Parasitology, v.120, p.91106, 2004.

CHAGAS, A.C.S.; OLIVEIRA, M.C.S.; FERNANDES, L.B.; MACHADO, R.; ESTEVES, S.N.; SALES, R.L.; JUNIOR, W.B. Ovinocultura: controle da verminose, mineralização, reprodução e cruzamentos na Embrapa Pecuária Sudeste. São Carlos, Embrapa Pecuária Sudeste, p. 44, 65, 2007.

COLES, G. C.; BAUER, C.; BORGSTEEDE, F. H. M.; GEERTS, S.; KLEI, T. R.; TAYLOR, M. A. WALLER, P. J. World Association for the Advancement of Veterinary Parasitology (W.A.A.V.P.) methods for detection of anthelmintic resistance in nematodes of Veterinary importance. Veterinary Parasitology, v.44, n. 1-2, p. 35-44, 1992.

COSTA, K.M.F.M., AHID, A.M.M., VIEIRA, L.S., VALE, A.M. e SOTOBLANCO, B. Efeitos do tratamento com closantel e ivermectina na carga parasitária, no perfil hematológico e bioquímico sérico e no grau de ovinos infectados com nematódeos. Pesquisa Veterinária Brasileira, v. 31 , p. 10751082, 2011.

CUNHA, E.A., SANTOS, L.E., BUENO, M.S. e VERÍSSIMO, C.J. Produção de ovinos para corte. Instituto de Zootecnia, p.141, 2007.

EMPRESA BRASILEIRA DE PESQUISA AGROPECUÁRIA EMBRAPA. Centro Nacional de Pesquisa de Solos. Sistema Brasileiro de Classificação de Solos. 2. Ed., 2006, p. 306.

FERREIRA, E. B., CAVALCANTI, P. P., NOGUEIRA,

A.

ExpDes.pt: Experimental package (Portuguese) $R$ package $R$ package version 1.1.2. Disponível em: http://CRAN.Rproject.org/package=ExpDes.pt, (2013). FORTES, F.S, KCOSTER, F.S, SCHAFER, A.S, BTER, D., BUZDTTI A., YOSHITANI U.Y, E MOLENTO M.B. Evalution of Resitance ind Selected Field Stratmof Haemochus contortus to ivermectina and moxidectin using the larval migration on agar test. Pesquisa Veterinária Brasileira, v. 33, n.2, p.183 - 187, 2013.

GEORGE, N., PERSAD, K., SAGAM, R., OFFIAH, V. N. ADESIYUN, A. A., HAREWOOD, W., LAMBIE, N., BASU, A., K. Efficacy of commonly used anthelmintic: first reporto f multiple drug resistance gastroinintestinal nematodes of sheep in Trinidad, Veterinary Parasitology, v. 183 , p. $194-197$, 2011.

GILL, B.S. Anthelmintic resistance in India. Veterinary Parasitology. v. 63, p.173-176, 1996.

GMC - Grupo Mercado Comum. Regulamento Técnico para Registros de Produtos Antiparasitários de Uso Veterinário. Decisão normativa 04/91, Resolução normativa 11/93. Mercosul, Resolução normativa 76/96, 1996. 
GORDON H.M.L. E WHITLOCK H.N. A new technique for counting nematode egg in sheep faeces. Journal of Scientific Industry Research. Austrália n.12(1), p.50-52, 1939.

\section{INSTITUTO BRASILEIRO DE} GEOGRAFIA E ESTATÍSTICA (IBGE), Anuário Estatístico 2013. Disponível em: http://www.ibge.gov.br. Acesso em: 07 de março de 2015.

JUSTO, R. V. Efeito do fungo nematófago Duddingtonia flagrans e da sazonalidade climática sobre 0 desenvolvimento e migração vertical de larvas infectantes de nematoides parasitos gastrintestinais de ovinos em pastagem de capim Aruana. Tese de Doutorado. Universidade Federal de Mato Grosso, 2014.

KAPLAN e VIDYASHANKAR. An inconventent Truch: Global Worming and Anthelmintic Resistante. Veterinary Parasitology, v.186, p.70 - 78, 2012.

KEITH R.K. The differentiation of the infective larvae of some common nematode parasites of cattle. Australian Veterinary Journal. n.1, p..223-235, 1953.

KÖSE M., KOZAN E., SEVIMLI F.K. e ESER M. The resistance of nematode parasites in sheep against anthelmintic drugs widely used in Western Turkey. Parasitologic Researt. v.101, p. 563567, 2007.

LIMA M.M., FARIAS M.P.O., ROMEIRO E.T., FERREIRA D.R. A. e ALVES L.C. e FAUSTINO M.A.G. Eficácia da moxidectina, ivermectina e albendazole contra helmintos gastrintestinais em propriedades de criação caprina e ovina no estado de Pernambuco. Ciências Animal Brasileira, v. 11, p. 94-100, 2010.

MADRUGA, M.S.; SOUSA, W. H.; ROSALES, M. D.; CUNHA, M. D. G.; RAMOS, J. L. F. Qualidade da carne de cordeiros Santa Inês terminados em diferentes dietas. Revista Brasileira de Zootecnia. v. 344, n.1, p. 309-315, 2005.

MELO, A.C., REIS, I.F., BEVILAQUA, C.M.L., VIEIRA, L.S., ECHEVARRIA, F.A.M. e MELO L.M. Nematódeos resistentes a anti-helmínticos em rebanhos de ovinos e caprinos do estado do Ceará, Brasil. Ciência Rural, v. 33, p. 339-344, 2003.

MENDIBURU, F. Agricolae: Statistical Procedures for Agricultural Research. R package version 1.2-1. Disponível em: http://CRAN.R-

project.org/package=agricolae, (2014).

MOLENTO, M.B. Resistência de helmintos em ovinos e caprinos. Revista Brasileira de Parasitologia, v. 13, n. 0, p. 82-86, 2004.

MORAES, F.R. Uso de marcadores imunológicos na avaliação da resposta imune dos ovinos à infecção natural por nematódeos e na seleção de animais resistentes às parasitoses. Dissertação (Mestrado em Ciências Veterinárias) Universidade Federal do Paraná, Curitiba, 2002. 194f.

$R$ CORE TEAM. R: A language and environment for statistical computing. $R$ Foundation for Statistical Computing, Vienna, Austria. Disponível em: http://www.R-project.org/, 2013.

ROBERTS F.H.S. e O'SULLIVAN J.P. Methods for egg counts and larval cultures for strongyles infesting the gastrointestinal tract of cattle. Australian Veterinary Journal, v.1, p. 99-102, 1950.

ROSALINSKI-MORAES F., MORETTO L.H., BRESOLIN W.S., GABRIELLI I., KAFER L., ZANCHET I.K., SONAGLIO F. e THOMAZ-SOCCOL. Resistência anti-helmíntica em rebanhos ovinos da região da Associação dos Municípios do Alto Irani (AMAI), Oeste de Santa Catarina. Ciência Animal Brasileira, v. 8, n.3, p. 559-565, 2007. 
SCZESNY-MORAES E.A, BIANCHIN I., SILVA K.F., CATTO J.B., HONER M.R. e PAIVA F. Resistência anti-helmíntica de nematopides gastrointestinais em ovinos, Mato Grosso do Sul. Pesquisa Veterinária Brasileira, v. 30, n.3, p.229236, 2010.

SIQUEIRA, E.R.; ROÇA, R. Q.; FERNANDES, S.; UEMI, A. Características sensoriais da carne de cordeiros das raças Hampshire Down, Santa Inês e mestiços Bergamácia $x$ Corriedale, abatidos com quatro distintos pesos. Revista Brasileira de Zootecnia, v. 31, n.3, p.1269-1272, 2002.

SOCCOL V.T., SOTOMAIOR C., SOUZA F.R. e CASTRO E.A. Occurrence of resistance to anthelmintics in sheep in Paraná state, Brazil Veterinary Record, v. 139, p. 421-422, 1996.

TRAVERSA D., PAOLETTI B., OTRANTO D. e MILLER J. First report of multiple drug resistance in trichostrongyles affecting sheep under field conditions in Italy. Parasitology Research, v. 101, p. 1713-1716, 2007. 